\title{
Simulation of Chemotaxis-based Sorting of Heterotypic Cell Populations
}

\author{
Manolya Eyiyurekli† \\ $\dagger$ Department of Computer Science \\ College of Engineering \\ Drexel University \\ Philadelphia, PA \\ \{me52, david\}@cs.drexel.edu
}

\author{
Peter I. Lelkes $\ddagger$ \\ David E. Breen† \\ $\ddagger$ School of Biomedical Engineering, \\ Science and Health Systems \\ Drexel University \\ Philadelphia, PA \\ pilelkes@drexel.edu
}

\begin{abstract}
Cell sorting is a fundamental process that is involved in early embryo development, tumerogenesis and morphogenesis. The sorting of heterotypic cell populations is produced by a variety of inter-cellular actions, e.g. differential adhesion and motility. We recently developed a software system that simulates chemotaxis-based cell aggregation in 2D. The model implemented within the system includes a variety of physiological cell behaviors, such as chemical diffusion/detection, motility, proliferation, adhesion and life cycle stages. In this paper we extend the model to simulate heterotypic cell sorting. This paper provides an overview of the chemotaxis-based aggregation model and describes the parameters and processes utilized to simulate the sorting of two different cell populations based on chemotactic interactions.
\end{abstract}

\section{INTRODUCTION}

The differential sorting of heterotypic cells resulting in the enclosure of one cell grouping by another leads to the organization of tissues during morphogenic processes such as embryogenesis, organogenesis and tumerogenesis. Determining the processes that influence cell sorting should provide insights into cellular development and may lead to deeper understanding of the mechanisms behind more complicated biological processes, such as tumor development. The processes involved in embryonic cell sorting during early development include differences in chemotaxis, cellular adhesiveness, cell surface contractility and cell motility [11]. We present a computational model that captures many of these behaviors and is able to produce in silico characteristic heterotypic cell sorting outcomes for a two-cell-type population.

We recently developed a computational model that successfully captures some of the cell behaviors that play important roles in 2-D cell aggregation [3], [4], [5]. A virtual cell in our model is designed as an independent, discrete unit with a set of physiologically relevant parameters and actions. Each cell is defined by its size, location, chemoattractant receptors, rates of chemoattractant release and response, age, life cycle stage, quiescent period, proliferation rate and number of attached cells. All cells are capable of releasing and sensing a chemoattractant chemical, moving, attaching to other cells, dividing, aging and dying. We have demonstrated that the model is capable of recreating a 24hour in vitro cell aggregation experiment. Here we have extended the computational model to simulate cell sorting among two-cell populations with different chemotactic and motility properties.

Computational simulations were conducted to identify the parameters most influential to cell sorting and their associated values. The final desired outcome consists of a central core of Type 1 (blue) cells surrounded by a layer of Type 2 (red) cells (see Figure 3). Once this result was produced a parametric study was performed to highlight the influence of the most important cell parameter, the probability that a Type 2 cell will respond to the Type 1 chemoattractant.

\section{OVERVIEW OF CHEMOTAXIS-BASED CELL AGGREGATION MODEL}

Within our computational model a cell's life cycle and behaviors are implemented as a set of actions that is performed during each time step of a simulation. A single aggregation simulation is comprised of a series of time steps. The actions taken by a cell at each of these time steps in our original aggregation simulations are outlined in Figure 1. Newly proliferated cells remain in a quiescent state for a user-defined period where they temporarily cannot divide. A quiescent cell is either separating from its sister cell or it is "waking up", i.e. beginning to respond to chemoattractant gradients. Based on the chemical fields produced by nearby cells a gradient is calculated and the cell responds to it by moving in the gradient direction. If cells collide, they attach to each other. A cell's age is incremented at each time step. If the cell is apoptotic (i.e. unattached after a specific age), it probabilistically determines if it should die. If the state of the complete environment is to be saved for visualization purposes, the cell emits and stores its chemical field in the chemoattractant array. If in the Active stage, the cell probabilistically determines if it is time to divide. If it divides, its and its daughter cell's age are set to zero, and they enter the quiescent stage.

All in-silico experiments represented in this paper contains 200 Type 1 and 200 Type 2 cells. All virtual cells live on a $500 \times 433 \mu \mathrm{m}$ grid representing a 2-D area of a Petri dish. Each cell has a radius of $6 \mu \mathrm{m}$. Chemoattractants are secreted from the cell's surface symmetrically and diffuse radially. The concentration of chemoattractant initially secreted by a single cell at its surface is $C_{0}$ molecules/ $\mu m^{2}\left(C_{0}=180\right.$ in our experiments). We assume that a constant chemical 


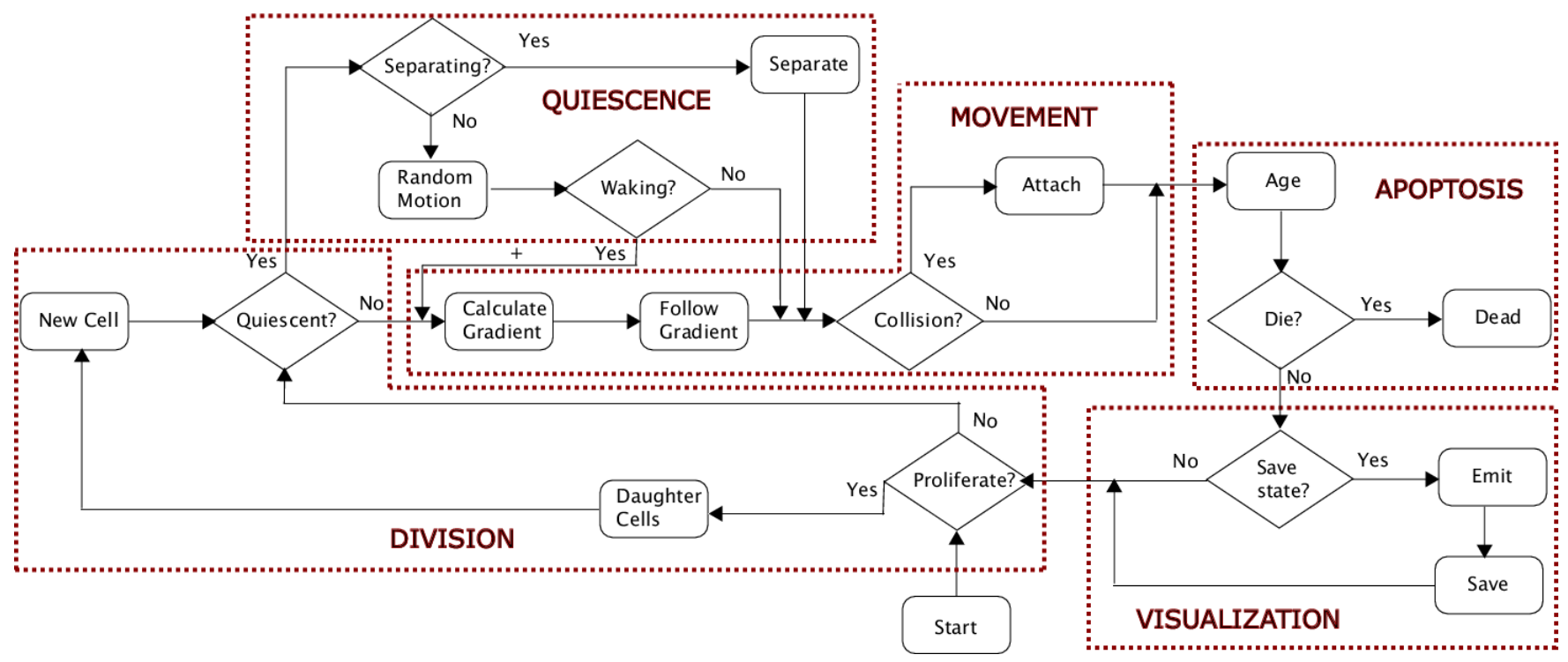

Fig. 1. Computational flow of cell aggregation simulation per time step per cell.

TABLE I

Model parameters by Cell type. $\lambda_{1}$ - Strength of Chemotactic Response to Chemical 1. $\lambda_{2}$ - StRength of Chemotactic Response to Chemical 2. $P_{1}$ - Probability of Responding to Chemical 1. $P_{2}$ - Probability of Responding to Chemical 2.

\begin{tabular}{|c|c|c|c|c|}
\hline Cell Type & $\lambda_{1}$ & $\lambda_{2}$ & $P_{1}$ & $P_{2}$ \\
\hline$T_{1}$ & 20.0 & 0.0 & 1.0 & 0.0 \\
\hline$T_{2}$ & 1.0 & 0.1 & 0.1 & 0.1 \\
\hline
\end{tabular}

concentration is maintained at the cell's surface, creating a static, circular chemical concentration field around each cell. Given this assumption, the chemoattractant concentration within the field drops off as $1 / r$, where $r$ is the distance from the cell surface [2],

$$
C(r)=\frac{C_{0}}{1+r}
$$

\section{CHEMOTAXIS-BASED CELL SORTING MODEL}

Several hypotheses have been proposed to explain cell sorting, for example differential adhesion [7], tensions that act along cell boundaries [1], differentials in chemotactic activity [9], intercellular repulsion [8] and cell motility [10].

We have chosen to focus on investigating the influence of chemotaxis and cell motility on heterotypic cell sorting. The computational model for studying cell sorting consists of a subset of features from the chemotaxis-based cell aggregation model. The cells in our sorting experiments do not attach to each other, divide or die, but they do age, and emit and respond to chemoattractant gradients. We observed that the fixed attachments formed between cells in our previous model limited the motion of the cells. While keeping the chemotactic interactions between the cells, we eliminated the fixed connections formed between cells; thus allowing cells to slide over one another. Additionally new features/parameters have been added to each cell, namely the excretion of a distinct chemoattractant, a chemotactic response rate and a probability of gradient following for each chemoattractant.

Our initial in-silico cell sorting experiments only contained two types of cell populations, $T_{1}$ and $T_{2}$. Each cell type emits a unique chemoattractant chemical, $C_{1}$ and $C_{2}$ respectively. Both types of cells can sense/respond to both types of chemicals and the strengths of these interactions are defined with parameters $\lambda_{1}$ and $\lambda_{2}$. A cell's velocity is proportional to the sum of the sensed, adjusted gradients,

$$
\text { Velocity }=\sum_{i=1}^{2} \lambda_{i} * \nabla C_{i} .
$$

Another parameter added in the sorting model is $P_{R i}$, the probability that a cell will follow the gradient of a specific chemoattractant $C_{i}$ during a simulation time step. If a cell does not respond to a gradient it takes a random step of 1 to $6 \mu \mathrm{ms}$. We found that $P_{R 1}$ for $T_{2}$ cells needed to be a function of time in order to produce the desired final result.

$$
\begin{gathered}
P_{R 1}= \begin{cases}P_{1} & 0 \leq A g e<T_{R} \\
F_{1}(\text { Age }) & T_{R} \leq A g e<2 * T_{R} \\
1.0 & \text { Age } \geq 2 * T_{R}\end{cases} \\
F_{1}(T)=\frac{1-P_{1}}{2} *\left(1-\cos \left(\pi *\left(T-T_{R}\right) / T_{R}\right)\right)+P_{1} .
\end{gathered}
$$

$T_{R}$ is the time at which a cell's chemotactic response increases. For $T_{2}$ cells, the probability of responding is initially $P_{1}$. At $T_{R}, P_{R 1}$ begins to increase with an interpolating cosine, reaching 1.0 by time $2 * T_{R}$. $P_{R i}$ is kept constant for the other interactions. These values are listed in Table I.

\section{RESULTS}

We performed numerous simulations with our computational model in order to determine which of its components most influence the creation of the sorting patterns presented in [6], [11]. We concluded that $\lambda_{i}$ and $P_{R i}$ have the greatest impact on cell sorting outcomes. The parameter values that 


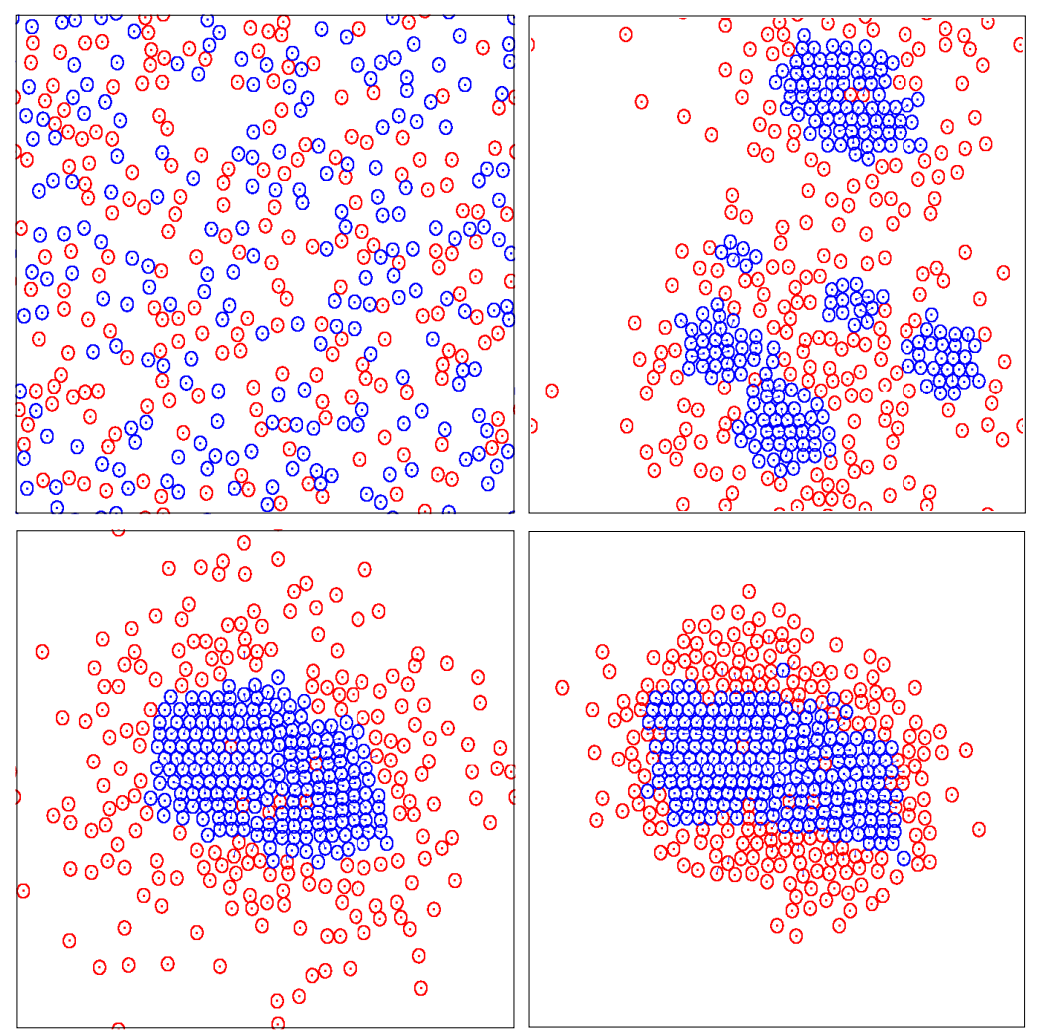

Fig. 2. Intermediate steps from an in silico cell sorting experiment. Initial state is top-left. Time increases left to right, top to bottom.

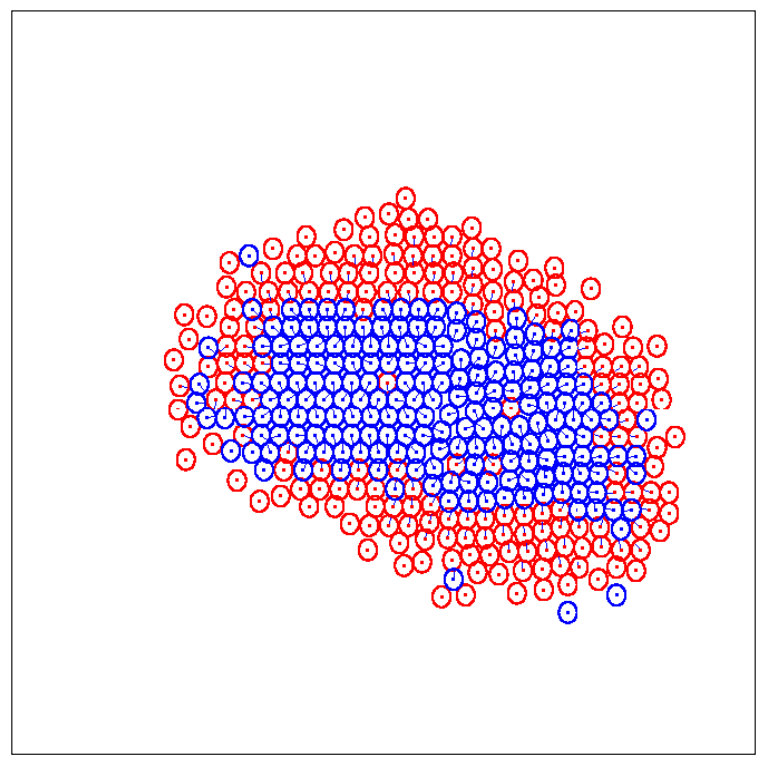

Fig. 3. Final result from a sorting simulation of a heterotypic mixture of two cell populations, type $T_{1}$ (blue) and type $T_{2}$ (red).

produce the desired result pictured in Figure 3 are listed in Table I. These values show that $T_{1}$ (blue) cells strongly respond to their own type of chemoattractant $\left(\lambda_{1}=20\right)$, but do not respond at all to $C_{2}\left(\lambda_{2}=0\right) . T_{2}$ cells respond weakly to $C_{1}\left(\lambda_{2}=1\right)$, and their response to their own chemoattractant $\left(C_{2}\right)$ is even weaker $\left(\lambda_{2}=0.1\right)$. Since $P_{i}$ is the probability that a cell will respond to chemoattractant concentration $C_{i}$, it can be seen that $T_{1}$ cells always respond to their own chemoattractant $\left(P_{1}=100 \%\right)$, and never respond to $C_{2}\left(P_{2}=0 \%\right)$. $T_{2}$ cells respond to their own chemoattractant $10 \%$ of the time. Initially $T_{2}$ cells respond to $C_{1} 10 \%$ of the time, but after $T_{R}$ their response rate ramps up to $100 \%$, as defined by Equation 3 .

As seen in Figure 2, the two cell populations are initially mixed. Since blue Type 1 cells strongly and always attract each other, they quickly form small aggregates, which then ultimately come together to form a single blue grouping. Red Type 2 cells move slower than blue cells and only respond to chemical gradients $10 \%$ of the time; thus producing a loosely packed "cloud" around the tightly packed blue Type 1 core (bottom left). After $T_{R}$ as the Type 2 cells become more strongly attracted to Type 1 cells, the Type 2 cells then form a tightly packed layer around the Type 1 cells (bottom right).

We also studied the effect of varying the $T_{2}$ cells' $P_{1}$ value on the structure of the final sorted cells. All $P_{i}$ values were kept constant during these simulations. The results for $P_{1}=$ $0,0.1,0.2$ and 1.0 are shown in Figure 4. When $P_{1}=0$ for Type 2 cells, they do not respond to $C_{1}$. Their response to $C_{2}$ is also weak. So as blue cells aggregate quickly, the red cells only show minimal signs of aggregating amongst themselves. See Figure 4 top left. Setting $P_{1}=0.1$ produces a result similar to Figure 2 bottom left. Our experiments showed that an increase in $P_{1}$ from 0.1 to 0.2 significantly changes the cell sorting behavior. Once this parameter is over $0.1, T_{2}$ cells aggregate more aggressively and begin to surround the $T_{1}$ cells. The $T_{2}$ cells' behavior interferes with the aggregation of the $T_{1}$ cells, and the $T_{1}$ cells remain in separate groups rather than aggregating into a single large 

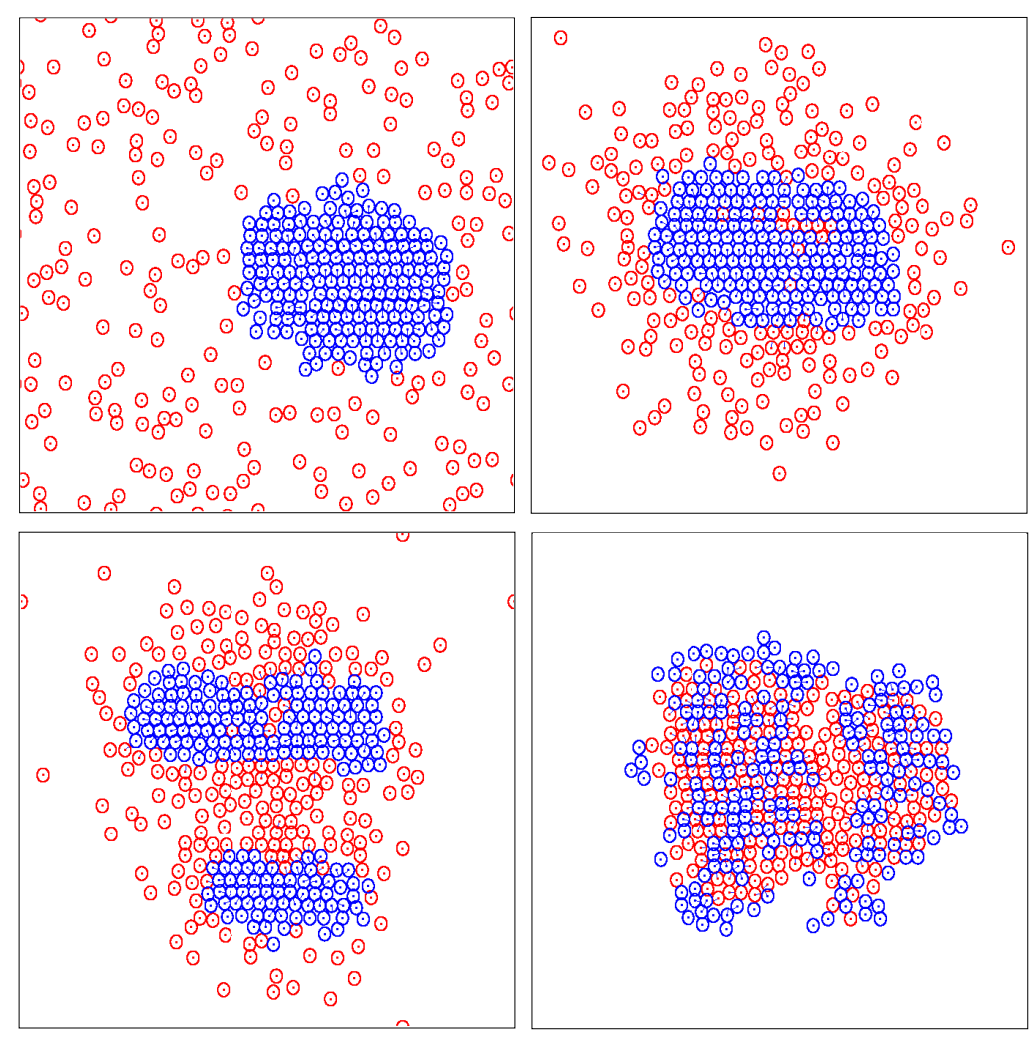

Fig. 4. Modifying $T_{2}$ 's probability of responding to chemoattractant $C_{1}$. Results for $P_{1}=0,0.1,0.2$ and 1.0. $T_{2}$ cells show little sign of aggregating when $P_{1}=0$ (top left). $P_{1}=0.1$ produces the same result as Figure 2 bottom left (top right). Increasing $P_{1}$ to 0.2 results in two smaller aggregates. Once the $T_{2}$ cells spread over the $T_{1}$ cells, the $T_{1}$ cells are prevented from aggregating further (bottom left). $T_{2}$ cells acts similar to $T_{1}$ cells and create an almost uniform distribution when $P_{1}=1$ (bottom right).

structure. See Figure 4 bottom left. A different sorting pattern is observed when the $T_{2}$ cells' $P_{R}=100 \%$. Since the $T_{2}$ cells follow the $C_{1}$ gradient all of the time they produce an aggregation behavior similar to the $T_{1}$ cells. Some sorting is evident since the blue Type 1 cells' speed is 20 times greater than the red Type 2 cells.

All simulations required approximately 1 CPU-hour of computation time on an Apple MacBook with Intel dual core $2.0 \mathrm{GHz}$ processor and $1 \mathrm{~GB}$ of RAM.

\section{CONCLUSION}

We have presented a 2-D chemotaxis-based computational model that successfully recreates heterotypic cell sorting behavior. By adjusting the parameters and processes of a chemotaxis-based cell aggregation model, we simulated the sorting of two cell populations with differing chemotactic and motility properties. Our in silico experiments identified the specific cell characteristics that affect chemotaxis-based cell sorting. The probability that a cell will follow a chemical gradient and the cell's speed in response to the gradient are the main factors that control cell sorting outcomes in our model.

\section{ACKNOWLEDGMENTS}

This research was funded by National Science Foundation grant CCF-0636323 and NASA grants NCC9-130 and NAG97-HEDS-02.

\section{REFERENCES}

[1] G. W. Brodland and H. H. Chen. The mechanics of heterotypic cell aggregates: insights from computer simulations. Journal of Biomechanical Engineering, 122:402-407, 2000.

[2] J. Crank. The Mathematics of Diffusion. Oxford University Press, Oxford, 2nd edition, 1975

[3] M. Eyiyurekli. A computational model of chemotaxis-based cell aggregation. Master's thesis, Drexel University, Philadelphia, PA, 2006.

[4] M. Eyiyurekli, P.I. Lelkes, and D.E. Breen. A computational system for investigating chemotaxis-based cell aggregation. In Proc. European Conference on Artificial Life, pages 1034-1049, 2007.

[5] M. Eyiyurekli, P. Manley, P.I. Lelkes, and D.E. Breen. A computational model of chemotaxis-based cell aggregation. 2007. submitted to BioSystems

[6] R. A. Foty, C. M. Pfleger, G. Forgacs, and M. S. Steinberg. Surface tensions of embryonic tissues predict their mutual envelopment behavior. Development, 122:1611-1620, 1996.

[7] F. Graner and J. A. Glazier. Simulation of biological cell sorting using a two-dimensional extended potts model. Physical Review Letters, 69:2013-2016, 1992.

[8] H. Honda and A. Mochizuki. Formation and maintenance of distinctive cell patterns by coexpression of membrane-bound ligands and their receptors. Developmental Dynamics, 223:180-192, 2002.

[9] T. Miura and K. Shiota. Tgfbeta2 acts as an "activator" molecule in reaction-diffusion model and is involved in cell sorting phenomenon in mouse limb micromass culture. Developmental Dynamics, 217:241249, 2000.

[10] G. Rogers and N. S. Goel. Computer simulation of cellular movements: Cell-sorting, cellular migration through a mass of cells and contact inhibition. Journal of Theoretical Biology, 71:141-166, 1978.

[11] M. S. Steinberg and M. Takeichi. Experimental specification of cell sorting, tissue spreading, and specific spatial patterning by quantitative differences in cadherin expression. Developmental Biology, 91:206209, 1994. 